\title{
Banana starch and molecular shear fragmentation dramatically increase structurally driven slowly digestible starch in fully gelatinized bread crumb
}

\author{
Laura Román ${ }^{1,4}$, Manuel Gómez ${ }^{1}$, Bruce R. Hamaker ${ }^{4}$, Mario M. Martinez ${ }^{2,3,4 *}$ \\ ${ }^{1}$ Food Technology Area. College of Agricultural Engineering, University of Valladolid, \\ 34004 Palencia, Spain. \\ ${ }^{2}$ School of Engineering, University of Guelph, Guelph, ON N1G 2W1, Canada. \\ ${ }^{3}$ Department of Food Science, University of Guelph, Guelph, ON N1G 2W1, Canada. \\ ${ }^{4}$ Whistler Center for Carbohydrate Research, Department of Food Science, Purdue \\ University, IN 47907, USA.
}

*Corresponding author e-mail: mario.martinez@uoguelph.ca;

\begin{abstract}
The role of native (NB) and extruded (EB) banana starch, and a 1:1 native:extruded banana starch composite (MB), in slowing down the starch digestibility of bread crumb and crust was investigated. During extrusion, the molecular weight of banana starch was reduced from $2.75 \times 10^{8}$ to $4.48 \times 10^{6} \mathrm{~g} / \mathrm{mol}$ (HPSEC-MALS-RI). Results showed a slowly digestible starch (SDS) increase from $1.09 \%$ (control) to 4.2, 6.6, and $7.76 \%$ in $\mathrm{NB}, \mathrm{MB}$ and EB crumbs (fully gelatinized), respectively. DSC data attributed this occurrence to the formation of supramolecular structures upon storage involving amylopectin branches (especially those from fragmented amylopectin in EB). The hedonic sensory test showed no differences in overall liking between MB, EB and control, validating feasibility of including banana in the formulation. For the first time, this study shows a molecular size reduction as a strategy to manufacture selected starches that result in highly gelatinized baked products rich in structurally driven SDS.
\end{abstract}

Keywords: banana starch; extrusion; digestion; retrogradation; gluten-free bread 


\section{Introduction}

Dietary glycemic carbohydrates that result in small excursions in postprandial plasma glucose and insulin concentrations are associated with improved insulin secretion and sensitivity and enhanced glycemic control (Vinoy, Laville, \& Feskens, 2016). On the other hand, recurrent postmeal high blood glucose levels have been linked to an increased risk of cardiovascular events and type two diabetes mellitus (Ceriello \& Colagiuri, 2008). Starch, the major polysaccharide related to postprandial glycaemia, is hydrolyzed by salivary and pancreatic $\alpha$-amylase in the mouth and small intestine of humans, and then to glucose by the mucosal brush border $\alpha$-glucosidases. The rate and extent to which starch is digested by these enzymes will greatly influence differences in the glycemic response of starch-based products. Since bread constitutes the highest proportion of carbohydrates (mainly starch) to the daily dietary intake in many parts of the world (Cust et al., 2009), successful strategies that decrease the digestibility of fully gelatinized starch might impact the prevalence of metabolic syndrome associated diseases.

In baked goods, the rate of starch digestibility has been correlated with the degree of starch gelatinization (Englyst, Vinoy, Englyst, \& Lang, 2003). The amorphous structure of gelatinized starch results in a greater availability of $\alpha$-amylase binding sites, which makes the substrate more susceptible to enzyme hydrolysis. Most of starches have been reported to gelatinize approximately in the range from 54 to $78{ }^{\circ} \mathrm{C}$ in excess of water $(\geq$ $20 \%$ water) (Vamadevan, Bertoft, \& Seetharaman, 2013). Therefore, considering that the moisture content of bread crumb is always $\geq 35 \%$ (Martinez, Roman, \& Gomez, 2018), where temperatures $\geq 70{ }^{\circ} \mathrm{C}$ are reached (Thorvaldsson \& Skjoldebran, 1998), a complete starch gelatinization in high moisture baked goods, such as bread, is expected to occur. In fact, Primo-Martin, van Nieuwenhuijzen, Hamer, and van Vliet (2007) and Martinez et al. (2018) reported a complete starch gelatinization in wheat bread crumb. Furthermore, Martinez et al. (2018) reported a complete starch gelatinization in candeal bread, also known as Fabiola or Bregado, with only 45\% dough hydration (close to the minimum amount of water possible to make bread).

Retrogradation is the recrystallization process of glycan chains in gelatinized starch, during which dispersed starch molecules begin to re-associate through $\mathrm{H}$-bonding. Albeit retrogradation has been reported as one reason for bread staling (Gray, \& BeMiller 2003), it is also known to result in a reduction of the rate and extension of starch digestion, depending on the main constituent involved. Thus, amylose double 
helices are known to be enzymatically resistant and yield resistant starch (RS) [Patel et al., 2017], whereas retrograded amylopectin has been attributed to the formation of slowly digestible starch (SDS) [Cui \& Oates, 1997; Zhang, Sofyan, M., \& Hamaker, 2008]. Based on previous evidence indicating that cereal starches containing high proportions of amylopectin with long chains (Benmoussa, Moldenhauer, \& Hamaker, 2007), or amylopectin with longer chains (Zhang, et al. 2008), result in a higher amount of slowly digestible starch in fully gelatinized material, Martinez, Li, Okoniewska, Mukherjee, Vellucci, and Hamaker (2018) investigated starch molecular structuredigestibility relationships in fully gelatinized starches. These authors reported for the first time two potential factors that may result in slowly digestible supramolecular structures during storage, namely: 1) double helices between A and B1 chains (unsubstituted and substituted short chains with a similar degree of polymerization, DP $<\sim 36$, respectively) of an average length $\geq 15.5$ Glucose Units (perhaps involving internal long chains) that also are prone to forming intermolecular associations and; 2) interactions of starch molecules with lower molecular size that may be more mobile and easily aligned during retrogradation. However, the effect of starch molecules with both significantly small size and long amylopectin chains resulting in structurally-driven slowly digestible starch has not been studied.

Banana starch has been reported to have amylopectin with small size (Yoo \& Jane, 2002) and a low proportion of short chains (Zhang \& Hamaker, 2012). It can be added into bakery products as purified starch or starch-predominant flour particles after simple liquid-solid extraction (Zhang, Whistler, BeMiller, \& Hamaker, 2005) or after simple processing involving drying and grinding (Segundo, Román, Gómez, \& Martínez, 2017) of banana pulp, respectively. When banana bunches arrive at central collection stations, bananas too small for shipping are removed, along with those that have damaged or spoiled areas. These rejected bananas are normally disposed of improperly and their successful industrial use would significantly reduce fruit food waste. Based on the aforementioned mechanistic understanding provided by Martinez et al. (2018), we hypothesized that: 1) banana starch, an untapped food by-product, would result in breads with a significant increase in slowly digestible starch and; 2) that the reduction of its molecular size through shear scission by means of extrusion (Zhang et al., 2015) would further slowdown the digestion rate of the starch glycemic fraction in breads. The objective of this study was, therefore, to identify a starch material that results in slowly digestible starch from fully gelatinized material. To do so, gluten-free breads served as 
an ideal high-moisture baked system (and therefore with extensive starch gelatinization) to investigate the effect of native and extruded banana starch on their in vitro starch digestion and physical and sensory properties. The starch digestion fractions and degree of starch gelatinization and retrogradation were analyzed in the crust and the crumb separately to provide insights about the spatial location and origin of slowly digestible starch.

\section{Materials and Methods}

\subsection{Materials}

Atlantic rice flour (9.58 \% moisture, $7.43 \%$ protein, $0.11 \% \mathrm{RS}, 12.49 \%$ SDS) was provided by Molendum Ingredients (Zamora, Spain), Miwon maize starch $(8.07 \%$ moisture, non-detected protein, $0.76 \%$ RS, $63.44 \%$ SDS) was purchased from Daesang Co. (Seoul, Korea) and banana starch from green lady finger bananas (6.30 \% moisture, $3.17 \%$ protein, $42.21 \%$ RS, $8.70 \%$ SDS) was purchased from Natural Evolution (Walkamin, Queensland, Australia). Starchy materials (including extruded banana flour) were characterized according to AACC methods (AACC, 2015) for moisture (4415.02), protein content (46-30.01) with a Leco TruSpec device (Leco, St. Joseph, MI, USA) and resistant starch content (32-40.01) using RS Megazyme assay kit. SDS was analyzed as detailed in section 2.2.5. Particle size was measured with a laser diffraction particle size analyzer (Mastersizer 3000, Malvern Instruments, Ltd., Worcestershire, UK). The mean diameter of equivalent volume $\mathrm{d}(4,3)$, which indicates the central point of the volume distribution of the particles, was recorded. The plot of the particle size distribution is included as Fig. 1A. Thermal properties were analyzed in triplicate as reported by Roman, Gomez, Li, Hamaker and Martinez (2017) using a differential scanning calorimeter Q-20 (TA instruments, Crawley, UK) equipped with a RCS 40 refrigerated cooling system (supplementary material 1 and 3). Furthermore, the pasting profile of the starchy materials (supplementary material 2) was obtained following the standard method 61-02.01 (AACC, 2015) with a Rapid Visco Analyser (RVA-4) (Perten Instruments Australia, Macquarie Park, Australia).

The ingredients used for bread making were VIVAPUR 4KM HPMC (Hydroxypropyl Methylcellulose, JRS, Rosenberg, Germany), Saf-Instant dry yeast (Lesaffre, Lille, France), salt (Union Salinera de España, Madrid, Spain), sucrose (Azucarera, AB, Madrid, Spain), Abrilsol sunflower oil (Ourense, Spain) and tap water. For performing starch in vitro digestion through the Englyst test, amyloglucosidase (EC 3.2.1.3) from 
Aspergillus niger, $300 \mathrm{U} / \mathrm{mL}$, pepsin (EC 3.4.23.1) from porcine stomach mucosa, 800$2500 \mathrm{U} / \mathrm{mg}$ solid, pancreatin from porcine pancreas, 8 x USP Specification, and guar gum were purchased from Sigma Chemical Co. (St. Louis, MO). Glucose assay reagents were from Megazyme International Ireland Ltd. (Wicklow, Ireland).

\subsection{Methods}

\subsubsection{Extrusion process}

The extrusion of banana starch (final moisture of $3.33 \%$ ) was performed in a Krupp Werner and Pfleiderer ZSK-25 twin screw extruder (Ramsey, NJ, USA) with corotating and closely intermeshing screws of $25 \mathrm{~mm}$ diameter and length to diameter (L/D) equal to 25 . The extruder was equipped with 5 barrel sections heated to 40,60, 90, 120 and $130 \pm 1{ }^{\circ} \mathrm{C}$ from the feeding to the die, respectively. The screw speed was maintained constant to $200 \mathrm{rpm}$. A combination of feeding, transporting, compression and mixing elements was used to provide a moderate-shear screw configuration that resulted in $330 \mathrm{KJ} / \mathrm{Kg}$ of specifical mechanical energy (SME). Feed input flow rate was $5 \mathrm{Kg} / \mathrm{h}$ with a water addition to obtain a total moisture content of $22 \%$. Banana starch was extruded through two circular dies of $4 \mathrm{~mm}$ diameter (each). Extruded banana starch was dried overnight at $55{ }^{\circ} \mathrm{C}$ by convection air and then ground with a pin mill. After milling, extruded banana starch $(3.29 \%$ protein, $0.84 \% \mathrm{RS}, 42.09 \%$ SDS $)$ contained a mean particle size $[\mathrm{d}(4,3)]$ of $299 \mu \mathrm{m}$, which was similar to the $\mathrm{d}(4,3)$ of native banana starch $(268 \mu \mathrm{m})$ due to the presence of particles formed by starch aggregates (Fig. 1A,B).

\subsubsection{Native and extruded banana starch molecular structure}

Starch structure of native banana starch was analyzed in triplicate using the method reported by Cave, Seabrook, Gidley, and Gilbert (2009) and Vilaplana, Hasjim, \& Gilbert, (2012) with minor modification as reported previously in Martinez et al. (2018). Analyses were performed using a size exclusion chromatography (SEC) system (Agilent 1260 series, Agilent Technologies, Waldbronn, Germany) equipped with a refractive index detector (RID, 1260 RID, Agilent, Agilent Technologies, Waldbronn, Germany). The length of short (A and B1) and long internal (B2 andB3, with average DP 42-48 and 69-75, respectively) amylopectin branches is denoted as $\mathrm{X}_{\mathrm{AP} 1}$ and $\mathrm{X}_{\mathrm{AP} 2}$, respectively, and the molar ratio of long to short amylopectin chains is represented as $\mathrm{h}_{\mathrm{Ap} 2 / \mathrm{Ap} 1}$. The amylose content of banana starch was determined from the SEC molecular size distribution of debranched starch (supplementary material 4) as the ratio of the area under the curve (AUC) of amylose branches to the AUC of overall amylopectin and 
amylose branches (Vilaplana et al., 2012). The chain length distribution of extruded banana starch was not analyzed since it has been reported not to be altered after extrusion (Zhang et al., 2015).

The molecular weight $(\mathrm{Mw})$ of banana starch molecules was measured in triplicate using a multi-angle light scattering detector (MALS, Dawn Heleos, Wyatt Technology, Santa Barbara, CA, USA) containing a K5-cell. $20 \mu \mathrm{L}$ of starch [previously dissolved in DMSO/LiBr as described in Martinez et al. (2018) for the analysis of the size of fully branched starch] were injected into GRAM 30 and 3000 columns (PSS GmbH, Mainz, Germany) connected in series $\left(0.3 \mathrm{~mL} / \mathrm{min}\right.$ at $\left.80^{\circ} \mathrm{C}\right)$. Data was analyzed with ASTRA software (version 4.72.03, Wyatt Technology Corporation, Goleta, CA, USA) and using the Berry second order plot procedure. This procedure involves an extrapolation of the function $\sqrt{ }\left(K^{*} c / R_{\theta}\right)$ to zero scattering angle $\theta$ [as $\left.\sin ^{2}(\theta / 2)\right]$. $K^{*}$ is a constant depending on the wavelength of the light and refractive index increment of the starch and $R_{\theta}$ is the 'Rayeligh excess ratio', which is a measure of the ratio of the intensity of excess light scattered compared to pure solvent at an angle $\theta$ to that of the incident light intensity (Harding, Adams, \& Gillis, 2016). The specific refractive index increment (dn/dc) was assumed to be $0.066 \mathrm{~mL} / \mathrm{g}$ as usually reported for starch dissolved in DMSO (Zhong, Yokoyama, Wang, \& Shoemaker, 2006) and the second viral coefficient $\left(\mathrm{A}_{2}\right)$ was assumed to be negligible (Yoo \& Jane, 2002).

\subsubsection{Dough preparation and bread-making}

Breads were made with water (105 g/100 g flour-starch mixture), instant dry yeast (3 $\mathrm{g} / 100 \mathrm{~g})$, salt (2 g/100 g), oil (6 g/100 g), HPMC (2 g/100 g) and white sugar (5 g/100 g). The base starchy source was comprised by a 1:1 mixture of maize starch and rice flour (control). Native banana starch (NB), extruded banana starch (EB) and a 1:1 mixture of native and extruded banana starches (MB) were used as a replacement of 20 $\%$ of the base starchy material. In all makings, water temperature was held between 20 and $22{ }^{\circ} \mathrm{C}$ for yeast dissolution before its incorporation. All the ingredients were mixed for $8 \mathrm{~min}$ at speed 2 with a flat beater (K45B) in a Kitchen Aid 5KSM150 mixer (Kitchen Aid, Michigan, USA). Subsequently, $150 \mathrm{~g}$ of dough were placed in small aluminum molds $(109 \times 159 \times 38 \mathrm{~mm})$ and fermented at $30^{\circ} \mathrm{C}$ and $90 \%$ relative humidity for $60 \mathrm{~min}$. After fermentation, doughs were baked in an electric modular oven for 40 $\min$ at $190{ }^{\circ} \mathrm{C}$. After baking, the loaves were demolded after $5 \mathrm{~min}$ and let to cool down for a total time of $60 \mathrm{~min}$ before their storage in sealed polyethylene bags at $20{ }^{\circ} \mathrm{C}$ for $24 \mathrm{~h}$. All the elaborations were prepared in duplicate. 


\subsubsection{Microstructural analysis of bread}

Bread and banana starch photomicrographs were taken with Quanta 200FEI (Hillsboro, Oregon, USA) environmental scanning electron microscope (ESEM). Photomicrographs were taken in high vacuum mode. Crumb and crust samples were pictured from a parallel slant (lengthwise section showing the thickness of the crust), whereas crust was also imaged from a perpendicular slant (supplementary material 5 and 6). In order to better assess crumb macrostructure, bread slices were pictured with a Canon EOS 1300D (Tokyo, Japan).

\subsubsection{Moisture content, starch thermal transitions and slowly digestible and resistant starch content in bread crumb and crust}

Crumb, taken from the center of a bread slice, and crust samples, obtained by scratching the bread surface, were analyzed for moisture content according to approved method 44-15.02 (AACC, 2015). The starch thermal transitions were measured as in Martinez et al. (2018), although results from the degree of starch gelatinization must be interpreted differently since, in the present study, a pre-gelatinized starch source is added into the recipe. Thus, DSC measures the reduction of the endothermal peak attributed to native amylopectin double helices between dough and bread (crumb or crust), which indicates the degree of starch gelatinization produced during baking $\left(\mathrm{DG}_{\mathrm{B}}\right)$. If all the starch added into the recipe was in a native state, the degree of starch gelatinization during baking would be equal to the percent of gelatinized starch in the final product (g gelatinized starch/100 g starch). However, in this case, results must be interpreted as a reduction of native starch (or an increase of gelatinized starch) during baking, which does not necessarily represent the final percent of gelatinized starch in the final product. DSC thermograms of the control dough and bread crumb and crust are reported as an example in supplementary material $3 . \mathrm{DG}_{\mathrm{B}}$ was calculated as:

$\mathrm{DG}_{\mathrm{B}}(\%)=\left(1-\frac{\Delta H D}{\Delta H B}\right) * 100$

where $\Delta \mathrm{H}_{\mathrm{D}}$ is the enthalpy of a portion of dough and $\Delta \mathrm{H}_{\mathrm{B}}$ is the enthalpy of crumb or crust samples, respectively.

The content of slowly digestible and resistant starch in flours/starches as well as in crumb and crust samples were analyzed as in Martinez et al. (2018), who followed the procedure described in Englyst, Kingman and Cummings (1992), and the AACCI approved method 32-40.01 (AACC, 2015) with the RS assay kit from Megazyme 
(Megazyme International, Wicklow, Ireland) for slowly digestible and resistant starch fractions, respectively. Each sample was analyzed in triplicate.

\subsubsection{Specific volume of breads and crumb texture}

Bread volume was determined using a laser sensor with the Volscan Profiler (Stable Micro Systems, Godalming, UK). The volume measurements were performed on three loaves of each elaboration. The specific volume was calculated as the ratio of bread volume to its mass. Crumb texture was measured after $24 \mathrm{~h}$ storage with a TA-XT2 texture analyzer (Stable Microsystems, Surrey, UK) equipped with the "Texture Expert" software. A 25-mm diameter cylindrical aluminum probe was used in a "Texture Profile Analysis" (TPA) double-compression test to penetrate up to $50 \%$ of the sample depth at a test speed of $5 \mathrm{~mm} / \mathrm{s}$, with a $30 \mathrm{~s}$ delay between the two compressions. Hardness $(\mathrm{g})$, cohesiveness, springiness and resilience were calculated from the TPA curve. Texture analyses were performed on two $30 \mathrm{~mm}$ central slices from two loaves from each elaboration.

\subsubsection{Consumer testing}

Hedonic sensory evaluation of gluten-free breads was conducted with 83 volunteers, between 16-65 years of age and from various socioeconomic backgrounds. Consumer test was conducted at a sensory laboratory in individual booths. Breads were assessed for their appearance, odor, flavor, texture, and overall liking on a nine-point hedonic scale. The scale ranged from "extremely like" to "extremely dislike" corresponding with highest and lowest scores of "9" and "1", respectively. Samples were presented on white plastic dishes coded with four-digit random numbers and served in random order. Water was available for rinsing. For the evaluation of bread appearance, a coded loaf of each bread type was presented. Samples were tested $24 \mathrm{~h}$ after baking.

\subsubsection{Statistical analysis}

Differences among results were studied by analysis of variance (one-way ANOVA). Fisher's least significant difference (LSD) was used to describe means with $95 \%$ confidence intervals. The statistical analysis was performed with the Statgraphics Centurion XVI software (Statpoint Technologies, Inc., Warrenton, USA).

\section{Results and Discussion}

\subsection{Moisture content, degree of starch gelatinization and amylopectin retrogradation in crumb and crust}


The moisture content ranged from 45.87 to $49.85 \%$ and from 18.40 to $21.21 \%$ in crumb and crust samples, respectively (Table 1). It is well known that, during baking, faster moisture transfer phenomena occur at the bread surface resulting in the development of a drying zone (Luyts et al., 2013), also known as crust, which will be a barrier for further heat and moisture transfer (Thorvaldsson \& Skjoldebran, 1998). In contrast, as soon as bread is removed from the oven, water rapidly migrates from the interior to the crust layer (Gorton, 2009). Results indicated a greater water migration from the crumb to the crust in EB, as seen by the significantly lower and higher moisture values in crumb and crust, respectively (Table 1). This might be attributed to two simultaneous factors: 1) a lower water retention capacity (loose bound water) of extruded banana starch (as suggested with the low pasting curve shown in supplementary material 2), as a consequence of starch fragmentation (Fig. 2) and/or; 2) a lower bread specific volume that may facilitate a faster transfer of water to the crust (i.e., shorter path for water to reach the bread surface).

The starch gelatinization in crumb during baking $\left(\mathrm{DG}_{\mathrm{B}}\right)$ was complete for all breads (Table 2). Conversely, $\mathrm{DG}_{\mathrm{B}}$ was limited in the crust, ranging from $5.56 \%$ in NB to 8.48 $\%$ in EB. The fast water evaporation suffered on the bread surface as the result of the high oven temperatures causes a rapid water depletion enough to hinder starch from gelatinization in the crust (Primo-Martín et al., 2007). Interestingly, NB crust displayed a lower $\mathrm{DG}_{\mathrm{B}}$ than the control, which suggests that the higher onset temperature of starch gelatinization $\left(\mathrm{T}_{\mathrm{o}}\right)$ of banana starch $\left(71.74{ }^{\circ} \mathrm{C}\right)$ compared to that from rice $\left(67.57{ }^{\circ} \mathrm{C}\right)$ and maize $\left(62.12{ }^{\circ} \mathrm{C}\right.$, supplementary material 1$)$ is enough to result in a significant reduction of starch gelatinization in the crust (even with a $20 \%$ replacement). On the other hand, the higher $\mathrm{DG}_{\mathrm{B}}$ in $\mathrm{EB}$ crust could be explained by the higher moisture content in the crust over the course of baking due to the higher water migration from the crumb to the crust as discussed before. This would result in higher availability of water for the starch to gelatinize.

During cooling and storage, amylopectin branches of gelatinized starch tend to reassociate with other amylose and amylopectin chains through the formation of double helices or aggregates of double helices (Klucinec \& Thompson, 2002). The melting of these structures can be detected by DSC (Martinez et al. 2018) and in this study, crumbs of breads stored for 1 day after baking exhibited the onset of this transition between 43 $\pm 1^{\circ} \mathrm{C}$ and $68 \pm 1{ }^{\circ} \mathrm{C}$ (supplementary material 3). The addition of banana starch resulted in an increase of the enthalpy for retrograded amylopectin in crumb (Table 2), although 
significant differences were only observed in EB and MB. HPSEC results revealed that banana amylopectin contains short chains (A and B1) and long internal chains (B2, B3, etc.) of an average length $\left(\mathrm{X}_{\mathrm{AP}}\right)$ of 17.0 and 42.1 Glucose Units (GU), respectively, and a molar ratio of long to short chains $\left(\mathrm{h}_{\mathrm{AP} 2 / \mathrm{hAP} 1}\right)$ of 0.73 (data not shown). These values are significantly higher than those from the maize and rice amylopectins reported by Martinez et al. (2018), with $\mathrm{X}_{\mathrm{AP} 1}, \mathrm{X}_{\mathrm{AP} 2}$ and $\mathrm{h}_{\mathrm{AP} 2 / \mathrm{hAP} 1}$ lower than $14.6 \mathrm{GU}, 39.8 \mathrm{GU}$ and 0.58. Longer amylopectin chains have been already reported to be more prone to form molecular interactions during retrogradation (Klucinec \& Thompson, 2002). What is more, Zhang and Hamaker (2012) reported a higher retrogradation rate of cooked banana starch compared to maize and potato starches, which was associated with the higher proportion of long chains of banana amylopectin. Interestingly, a molecular size reduction of banana amylopectin from $2.75 \times 10^{8}$ to $4.48 \times 10^{6} \mathrm{~g} / \mathrm{mol}$ during extrusion (Fig. 2), led to a higher potential of extruded banana starch to form molecular interactions during retrogradation, which may be the result of a higher mobility and alignment properties. This occurrence was already observed by Martinez et al. (2018) in acid-converted maize starch with extensive amylopectin hydrolysis. It is worth mentioning that no significant differences in amylose content between banana and maize starches were found $(\sim 30 \%)$, although rice starch exhibited slightly lower amylose ratio (18\%, data not shown).

Unlike crumb samples, no endothermic peak attributed to retrograded amylopectin was detected in crust samples, which is likely ascribed to the lower moisture content and minimum amount of gelatinized starch in the crust.

\subsection{Physical properties of breads}

Crumb porosity and microstructure images (Fig. 3 and supplementary material 5) revealed slight differences in the crumb pore distribution of the breads. The gluten-free crumb consists of a porous and resilient HPMC-starch matrix that encloses, in honeycomb fashion, minute gas cells, making up the loaf volume. Control, NB and MB breads possessed a fine and closely-packed pore distribution, whereas EB bread crumb presented larger and heterogeneous cells. Carrillo-Navas et al. (2016) found a more heterogeneous crumb microstructure of (larger and heterogeneous gas cells) of bread containing gelatinized flour. This occurrence could be explained based on the thicker cell walls observed through SEM in MB and EB crumbs (supplementary material 5), which has been previously reported to result in coarser cell structures (Gorton, 2009). 
The specific volume of breads and textural properties of crumbs are shown in Table 1. Interestingly, the inclusion of native banana starch brought about breads with higher specific volume (Table 1 and Fig. 3), effect that was similarly reported by Sarawong, Gutiérrez, Berghofer, and Schoenlechner (2014) with inclusion of up to $25 \%$ of green plantain flour. It is well-known that during the first steps of baking, the expansion of gas cells embedded in the dough (i.e., oven rise) occurs until, when reaching a certain temperature $\left(60-90^{\circ} \mathrm{C}\right)$, the hydrated starch gelatinizes leading to crumb setting (LeBail et al., 2011). Therefore, the higher onset of gelatinization $\left(T_{0}\right)$ of banana starch (supplementary material 1) compared to the basic starch source (rice flour and maize starch) could give the dough extra time for expansion before crumb settling, as Gorton (2009) suggested to occur with other starches. This would also entail a greater integrity of banana starch granules during prolonged heating (Zhang et al., 2005), which would reinforce the continuous phase (starch-hydrocolloid matrix) surrounding the gas cells (Martinez \& Gomez, 2017; Roman, de la Cal, Gomez, \& Martinez 2018). On the other hand, the drop in the specific volume with the addition of extruded banana starch could be due to the slightly higher cold viscosity property of gelatinized starch compared to native counterparts, as observed by Martinez, Oliete, Román, and Gómez (2014) incorporating extruded rice flour in gluten-free breads.. A higher cold viscosity of extruded banana starch would increase dough consistency that hinders gas expansion, and therefore, limits dough development (Carrillo-Navas et al., 2016). Interestingly, in this work we further showed that a 1:1 native to extruded banana starch composite diminishes the negative effects of the presence of gelatinized starch in the dough, attaining breads with slightly lower specific volume than control but greater than EB.

The texture parameters of bread are presented in Table 1. A higher hardness was found when decreasing the specific volume of the gluten-free breads. An inverse correlation of bread specific volume with its hardness has been reported previously in several studies (Martinez, et. al. 2018; Pongjaruvat, Methacanon, Seetapan, Fuongfuchat, \& Gamonpilas, 2014). This higher resistance to crumb deformation has been related to the lower cell density (number of cells per area) and more compact structure in low volume breads. Furthermore, the lower moisture content of EB (Luyts et al., 2013) and the thicker cell walls of EB and MB crumbs (Carrillo-Navas et al., 2016; Martinez et al., 2014; Pongjaruvat et al., 2014) may have also accounted for its increased hardness. In general, an increase in hardness tends to be accompanied by a decrease in crumb elasticity and cohesiveness (Martínez, et al., 2018; Pongjaruvat et al., 2014). Although 
no significant differences were found for the springiness of any of crumb samples, more resilient and cohesive breads were evident when including native banana in the formula, which likewise could support the idea that banana starch help reinforce the dough matrix. Conversely, the inclusion of extruded banana starch resulted in less cohesive and resilient breads compared to the native banana counterpart, but with small differences with the control sample. In any case, the negative effect of extruded banana on crumb texture was minimized when mixed with its native counterpart.

\subsection{Starch digestibility in bread crumb and crust}

Starch digestion fractions corresponding to slowly digestible starch (SDS) and resistant starch (RS) were determined according to the Englyst time-based classification (Englyst et al., 1992) and shown in Fig. 4 as bread basis (b. b.). Control gluten-free bread crumb (Fig. 4) exhibited low SDS content (1.09\%), which is in agreement with results from multiple studies with breads (Martinez et al. 2018; Lau, Soong, Zhou, \& Henry, 2015) and may be expected due to the complete starch gelatinization in the crumb (Table 2). SDS significantly increased with the incorporation of banana starch and, especially, of extruded banana starch. Martinez et al. (2018) recently reported that amylopectin with a short chain population of branches of an average length higher than 15.5 GU may result in the formation of slowly digestible supramolecular structures from fully gelatinized material during retrogradation. In the present work, banana amylopectin short branches exhibited an average length of 17.0 GU that were involved in molecular interactions during retrogradation, as indicated by the higher enthalpy of retrograded enthalpy in banana containing crumbs (Table 2). These supramolecular assemblies have been reported to be slowly digested by different authors (Zhang, et al. 2008; Martinez et al. 2018). In this work, banana amylopectin also showed a higher proportion of long chains $\left(\mathrm{h}_{\mathrm{AP} 2 / \mathrm{hAP} 1}=0.73\right)$, structural feature that has been previously reported to result in faster retrogradation on cooling than other amylopectins and lead to retrograded starch less accessible to amylase digestion (Zhang and Hamaker 2012). Secondarily, other mechanical properties at macroscopic level, such as crumb porosity, hardness and resiliency, may have had an influence on starch bioaccesibility during in vitro digestion. Control and NB presented similar crumb porosity and specific volume and NB crumb was softer. Therefore, the higher cohesiveness of NB crumb would be the only contributor at reducing crumb breakdown during digestion, which would not coincide with the trend reported by Martinez et al. (2018) showing higher SDS in less cohesive bread crumb. 
As hypothesized in the present study, extruded banana starch resulted in a further increase of SDS in the crumb. Interestingly, a fivefold improvement in SDS performance (from 1.09 to $7.76 \%$ ) was attained with a $20 \%$ replacement of the base starchy ingredient by extruded banana starch. Martinez et al. (2018) reported that a molecular size reduction of amylopectin through acid-hydrolysis increased its propensity to re-associate during storage and resulted in slowly digestible supramolecular structures. Here, this occurrence is also demonstrated with shear molecular fragmentation during extrusion according to the higher enthalpy of retrograded amylopectin in MB and EB crumbs. Smaller amylopectin molecules could be more mobile and have better alignment properties during retrogradation. Added to that, $\mathrm{MB}$ and $\mathrm{EB}$ crumbs were harder and more compact, which may result in a higher resistance to physical breakdown during digestion and, therefore, in limitations of digestive enzymes to access the "entrapped" starch (Martinez, et al., 2018).

The amount of resistant starch in the crumb ranged from 1.45 to $1.89 \%$ (g/100 g bread), which should be considered low to promote any beneficial physiological implication. An enhancement in the RS content of the crumb was visible with the incorporation of banana starch, especially in MB and EB samples. Similarly, Sarawong et al. (2014) found a significant higher content of RS (up to $2.3 \%$ ) in gluten-free breads with the addition of $15-35 \%$ native banana flour. These authors related this event to the higher initial amounts of RS in the initial flour (RS2), although the degree of starch gelatinization and the spatial location of RS (crumb or crust) were not reported. In all our samples, since starch was completely gelatinized (Table 2), short-term retrogradation upon cooling and storage, mainly involving the re-association of amylose chains that are enzymatically resistant (RS3) [Patel et al., 2017], is expected to be the main precursor for the RS increase (Englyst, et al., 1992). In fact, banana starch presents an amylose content of $30 \%$ (relatively higher than that of the rice-maize base mixture). The native banana starch provided by the manufacturer contained $42.21 \%$ RS (in the form of RS2), however attention must be paid when this ingredient is incorporated into foods that will suffer hydrothermal processing with enough amount of water for starch gelatinization.

Crust samples presented significantly higher SDS (25.30-34.17\%) and relatively higher RS (0.26-5.66 \%) than crumb counterparts, which agrees with previous works (Martinez, et al. 2018) and was attributed to the significantly lower degree of gelatinization of bread crust compared to crumb (Table 2). Surprisingly, only the 
inclusion of native banana starch gave rise to a significant increase in SDS of bread crust, which could derive from the higher amount of ungelatinized maize starch, an inherently perfect source of SDS $(\sim 50 \%)$, resulting from the lower starch gelatinization in NB (Table 2). Added to that, part of the RS2 of native banana starch, which is relative fragile under cooking conditions (even after 10 min cooking), could have been converted into RDS and SDS (Bi et al., 2017).

Results also showed that a $20 \%$ replacement with native banana starch brought about the highest RS in the crust (5.66\%), which seems logical regarding the inherent higher resistance of banana starch in its native granular state (RS of 42.21\%). It is worth mentioning that the inclusion of extruded banana starch also resulted in a small but significant increase in RS compared to control crust (from 0.26 to $0.72 \%$ ), which could comprise RS3 (retrograded amylose) and RS5 (complexation of banana amylose with free lipids during extrusion) inherently developed in the raw ingredient during extrusion and upon cooling (Camire, Camire, \& Krumhar, 1990). In fact, extruded banana starch possessed higher RS than the combination of rice and maize flours.

\subsection{Sensory evaluation}

A hedonic sensory test was conducted to evaluate the feasibility of using banana starch as a nutritional improver from the carbohydrate standpoint (Table 1). Interestingly, the inclusion of native and extruded banana starch generally resulted in gluten-free breads with improved odor and flavor (the latter especially improved with extruded banana) compared with control bread. Visual appearance exhibited the greatest differences, highlighting a significantly lower rating with the use of extruded flour (EB), effect that was minimized when mixing this flour with the native counterpart (MB). We believe that it might be related to the lower specific volume of MB and EB compared to NB and control bread (as previously seen in Fig. 3 and Table 1), rather than to differences in color. In fact, some of the panelists indicated their preference for banana-based breads due to their darker color. Differences in crumb color (shown in Fig. 3) should be explained based on the darker brown color of banana starch compared with the whitish rice flour and maize starch (data not shown), as Segundo et al. (2017) already pointed out for wheat cakes enriched with banana flour. On the other hand, in bread crust, alongside the natural color of the starch material, the higher amounts of simple sugars in banana flour (Emaga, Andrianaivo, Wathelet, Tchango, \& Paquot, 2007) also significantly darken crust color. Furthermore, starch dextrinization during extrusion results in a subsequent formation of reducing sugars (Camire, et al., 1990) that 
participate in Maillard and caramelization reactions on the hot bread surface during baking (Gorton, 2009). Therefore, a greater color of EB crusts should be expected. Regarding the consumer perception for bread texture, a negative correlation with crumb hardness instrumentally measured was evident (Table 1), which was discussed in section 3.2 .

Despite the lower scores of EB for appearance and texture, interestingly some panelists expressed their preference for extruded banana containing breads due to their more pleasant flavor. In fact, EB bread was the best valuated in terms of flavor, which may have had an important weight at reducing the differences of overall liking. Specifically, differences for overall liking between EB and NB (the best-scored one) were not greater than 0.5 . The enhancement in the scores for odor and flavor with the inclusion of $20 \%$ banana starch are undoubtedly a positive indication of the feasibility of including banana in the formulation of gluten-free breads without declining their acceptability. In fact, in this study we attained breads with $30 \%$ replacement by native banana starch and a 1:1 native to extruded banana starch composite with acceptable physical attributes. However, $30 \%$ replacement was not chosen due to the flat bread (very low volume) with solely extruded banana starch.

Differences in bread appearance should be taken into account when including banana starch into the formulation, although this score can be modulated by increasing fermentation time or adjusting the water content (dough hydration) in the formulation in order to increase the specific volume of the loaves.

\section{Conclusions}

In this research work, we present a successful strategy to slow down the digestion rate of the fully gelatinized starch present in baked goods by the manipulation of the starch molecular structure. A and B1 chains of banana starch, with an average length of 17.0 GU, re-associated upon cooling (perhaps also with long internal amylopectin chains) forming slowly digestible supramolecular structures. This study also shows, for the first time, that the propensity of amylopectin molecules to form structurally driven SDS is further improved by a reduction of their molecular size by means of extrusion (a clean and cost-effective technology). Results of this work are also expected to contribute to the improvement of the sustainability of food systems and increasing local and global food availability. Banana pulp is an untapped food by-product and, in this work, we demonstrate its enormous nutritional potential with a successful application. 


\section{Acknowledgments}

L. R. and M. G acknowledge the financial support from the European Regional Development Fund (FEDER) and the Spanish Ministry of Economy and Competitiveness (Project AGL2014-52928-C2). L. R. would like to thank the University of Valladolid for her pre-doctoral fellowship. All authors are grateful to Molendum Ingredients for supplying the rice flour.

\section{References}

AACC (2015). Approved methods of the American Association of Cereal Chemists, Methods 44-15.02 (moisture), 32-40.01 (resistant starch), 46-30.01 (protein), 61-02.01 (rapid visco analysis), 11th ed. American Association of Cereal Chemists, St. Paul, Minnesota.

Benmoussa, M., Moldenhauer, K. A., \& Hamaker, B. R. (2007). Rice amylopectin fine structure variability affects starch digestion properties. Journal of agricultural and food chemistry, 55, 1475-1479.

Camire, M. E., Camire, A., \& Krumhar, K. (1990). Chemical and nutritional changes in food during extrusion. Critical Reviews in Foods Science and Nutrition, 29, 35-57.

Cave, R. A., Seabrook, S. A., Gidley, M. J., \& Gilbert, R. G. (2009). Characterization of starch by size-exclusion chromatography: the limitations imposed by shear scission. Biomacromolecules, 10, 2245-2253.

Carrillo-Navas, H., Guadarrama-Lezama, A. Y., Vernon-Carter, E. J., García-Díaz, S., Reyes, I., \& Alvarez-Ramírez, J. (2016). Effect of gelatinized flour fraction on thermal and rheological properties of wheat-based dough and bread. Journal of food science and technology, 53, 3996-4006.

Ceriello, A., \& Colagiuri, S. (2008). International Diabetes Federation guideline for management of postmeal glucose: a review of recommendations. Diabetic Medicine, 25, 1151-1156.

Cui, R., \& Oates, C. G. (1997). The effect of retrogradation on enzyme susceptibility of sago starch. Carbohydrate Polymers, 32, 65-72.

Cust, A. E., Skilton, M. R., van Bakel, M. M., Halkjaer, J., Olsen, A., Agnoli, C., et al. (2009). Total dietary carbohydrate, sugar, starch and fibre intakes in the European Prospective Investigation into Cancer and Nutrition. European Journal of Clinical Nutrition, 63, S37-S60. 
Emaga, T. H., Andrianaivo, R. H., Wathelet, B., Tchango, J. T., \& Paquot, M. (2007). Effects of the stage of maturation and varieties on the chemical composition of banana and plantain peels. Food Chemistry, 103, 590-600.

Englyst, K. N., Vinoy, S., Englyst, H. N., \& Lang, V. (2003). Glycaemic index of cereal products explained by their content of rapidly and slowly available glucose. British Journal of Nutrition, 89, 329-339.

Englyst, H. N., Kingman, S. M., \& Cummings, J. H. (1992). Classification and measurement of nutritionally important starch fractions. European Journal of Clinical Nutrition, 46, S33-S50.

Bi, Y., Zhang, Y., Jiang, H., Hong, Y., Gu, Z., Cheng, L., ... \& Li, C. (2017). Molecular structure and digestibility of banana flour and starch. Food Hydrocolloids, 72, 219-227. Gray, J. A., \& Bemiller, J. N. (2003). Bread staling: molecular basis and control. Comprehensive Reviews in Food Science and Food Safety, 2, 1-21.

Gorton, L. A. (2009) Fundamental Bakery Dough Processes. In E. J. Pyler \& L. A. Gorton (Eds.), Baking Science \& Technology Fourth Edition. Volume II: Formulation \& Products (pp. 1-136). Sosland Publishing Company, Kansas City, MO.

Harding, S. E., Adams, G. G., \& Gillis, R. B. (2016). Molecular weight analysis of starches: Which technique?. Starch-Stärke, 68, 846-853.

Klucinec, J., \& Thompson, D. (2002). Amylopectin nature and amylose-to-amylopectin ratio as influences on the behavior of gels of dispersed starch. Cereal Chemistry, 79, 2435.

Lau, E., Soong, Y. Y., Zhou, W., \& Henry, J. (2015). Can bread processing conditions alter glycaemic response? Food Chemistry, 173, 250-256.

Le-Bail, A., Leray, D., Lucas, T., Mariani, S., Mottollese, G., \& Jury, V. (2011). Influence of the amount of steaming during baking on the kinetic of heating and on selected quality attributes of bread. Journal of Food Engineering, 105, 379-385.

Luyts, A., Wilderjans, E., Haesendonck, I. V., Brijs, K., Courtin, C. M., \& Delcour, J. A. (2013). Relative importance of moisture migration and amylopectin retrogradation for pound cake crumb firming. Journal of Food Engineering, 108, 128-134.

Martinez, M. M., Li, C., Okoniewska, M. Mukherjee, N., Vellucci, D., \& Hamaker, B. R. (2018). Structure-digestion relationships of fully gelatinized starch: a novel approach to slow down the starch digestibility in hydrothermally-treated foods. Carbohydrate Polymers, 197, 531-539. 
Martinez, M. M., \& Gómez, M. (2017). Rheological and microstructural evolution of the most common gluten-free flours and starches during bread fermentation and baking. Journal of Food Engineering, 197, 78-86.

Martinez, M. M., Roman, L., \& Gómez, M. (2018). Implications of hydration depletion in the in vitro starch digestibility of white bread crumb and crust. Food Chemistry, 239, 295-303.

Martinez, M. M., Oliete, B., Román, L., \& Gómez, M. (2014). Influence of the addition of extruded flours on rice bread quality. Journal of Food Quality, 37, 83-94.

Patel, H., Royall, P. G., Gaisford, S., Williams, G. R., Edwards, C. H., Warren, F. J., ... \& Butterworth, P. J. (2017). Structural and enzyme kinetic studies of retrograded starch: Inhibition of $\alpha$-amylase and consequences for intestinal digestion of starch. Carbohydrate Polymers, 164, 154-161.

Pongjaruvat, W., Methacanon, P., Seetapan, N., Fuongfuchat, A., \& Gamonpilas, C. (2014). Influence of pregelatinised tapioca starch and transglutaminase on dough rheology and quality of gluten-free jasmine rice breads. Food Hydrocolloids, 36, 143 150.

Primo-Martín, C., van Nieuwenhuijzen, N. H., Hamer, R. J., \& van Vliet, T. (2007). Crystallinity changes in wheat starch during the bread-making process: Starch crystallinity in the bread crust. Journal of Cereal Science, 45, 219-226.

Roman, L., Gomez, M., Li, C., Hamaker, B.R., \& Martinez, M.M. (2017). Biophysical features of cereal endosperm that decrease starch digestibility. Carbohydrate Polymers, $165,180-188$.

Roman, L., de la Cal, E., Gomez, M., \& Martinez, M. M. (2018). Specific ratio of A- to B-type wheat starch granules improves the quality of gluten-free breads: optimizing dough viscosity and Pickering stabilization. Food Hydrocolloids. https://doi.org/10.1016/j.foodhyd.2018.04.034

Sarawong, C., Gutiérrez, Z. R., Berghofer, E., \& Schoenlechner, R. (2014). Effect of green plantain flour addition to gluten-free bread on functional bread properties and resistant starch content. International Journal of Food Science and Technology, 49, 1825-1833.

Segundo, C., Román, L., Gómez, M., \& Martínez, M. M. (2017). Mechanically fractionated flour isolated from green bananas (M. cavendishii var. nanica) as a tool to increase the dietary fiber and phytochemical bioactivity of layer and sponge cakes. Food Chemistry, 219, 240-248. 
Thorvaldsson, K., \& Skjöldebrand, C. (1998). Water diffusion in bread during baking. LWT-Food Science and Technology, 31, 658-663.

Vamadevan, V., Bertoft, E., \& Seetharaman, K. (2013). On the importance of organization of glucan chains on thermal properties of starch. Carbohydrate Polymers, 92, 1653-1659.

Vilaplana, F., Hasjim, J., \& Gilbert, R. G. (2012). Amylose content in starches: Towards optimal definition and validating experimental methods. Carbohydrate Polymers, 88, 103-111.

Vinoy, S., Laville, M., \& Feskens, E. J. (2016). Slow-release carbohydrates: growing evidence on metabolic responses and public health interest. Summary of the symposium held at the 12th European Nutrition Conference (FENS 2015). Food and Nutrition Research, 60, 31662.

Yoo, S. H., \& Jane, J. L. (2002). Molecular weights and gyration radii of amylopectins determined by high-performance size-exclusion chromatography equipped with multiangle laser-light scattering and refractive index detectors. Carbohydrate Polymers, 49, 307-314.

Zhang, G., Sofyan, M., \& Hamaker, B. R. (2008). Slowly digestible state of starch: mechanism of slow digestion property of gelatinized maize starch. Journal of Agricultural and Food Chemistry, 56, 4695-4702.

Zhang, P., \& Hamaker, B. R. (2012). Banana starch structure and digestibility. Carbohydrate polymers, $87,1552-1558$.

Zhang, P., Whistler, R. L., BeMiller, J. N., \& Hamaker, B. R. (2005). Banana starch: production, physicochemical properties, and digestibility-a review. Carbohydrate Polymers, 59, 443-458.

Zhang, B., Dhital, S., Flanagan, B. M., Luckman, P., Halley, P. J., \& Gidley, M. J. (2015). Extrusion induced low-order starch matrices: Enzymic hydrolysis and structure. Carbohydrate Polymers, 134, 485-496.

Zhong, F., Yokoyama, W., Wang, Q., \& Shoemaker, C. F. (2006). Rice starch, amylopectin, and amylose: molecular weight and solubility in dimethyl sulfoxide-based solvents. Journal of Agricultural and Food Chemistry, 54(6), 2320-2326. 
Table 1. Effect of native and extruded banana starch on physical properties of breads

\begin{tabular}{|c|c|c|c|c|c|c|c|c|c|c|c|c|}
\hline & Specific & & & & & Crumb & & & & & & \\
\hline Sample & $\begin{array}{l}\text { volume } \\
(\mathrm{mL} / \mathrm{g})\end{array}$ & Hardness $(\mathrm{g})$ & Springiness & Cohesiveness & Resilience & $\begin{array}{l}\text { moisture } \\
(\mathrm{g} / 100 \mathrm{~g})\end{array}$ & $(\mathrm{g} / 100 \mathrm{~g})$ & Appearance & Odor & Flavor & Texture & $\begin{array}{l}\text { Overall } \\
\text { liking }\end{array}$ \\
\hline Control & $4.29 c \pm 0.15$ & $622 b \pm 79$ & $0.99 a \pm 0.02$ & $0.38 b \pm 0.02$ & $0.16 b \pm 0.01$ & $49.36 b \pm 0.12$ & $18.95 a \pm 0.01$ & $6.6 \mathrm{c} \pm 1.4$ & $5.5 a \pm 1.6$ & $4.9 \mathrm{a} \pm 1.7$ & $5.4 \mathrm{~b} \pm 1.8$ & $5.5 \mathrm{ab} \pm 1.5$ \\
\hline Native Banana & $5.34 \mathrm{~d} \pm 0.13$ & $304 \mathrm{a} \pm 18$ & $0.99 a \pm 0.05$ & $0.45 c \pm 0.02$ & $0.21 c \pm 0.02$ & $49.85 c \pm 0.17$ & $18.96 a \pm 0.06$ & $6.5 c \pm 1.4$ & $6.0 \mathrm{~b} \pm 1.4$ & $5.3 \mathrm{ab} \pm 1.6$ & $5.8 b \pm 1.6$ & $6.0 \mathrm{~b} \pm 1.4$ \\
\hline Mixture Banana & $3.57 \mathrm{~b} \pm 0.07$ & $1051 \mathrm{c} \pm 146$ & $1.00 \mathrm{a} \pm 0.00$ & $0.36 \mathrm{ab} \pm 0.02$ & $0.14 \mathrm{a} \pm 0.01$ & $48.90 \mathrm{~b} \pm 0.01$ & $18.40 \mathrm{a} \pm 0.09$ & $5.9 b \pm 1.6$ & $6.1 b \pm 1.4$ & $5.1 \mathrm{ab} \pm 1.6$ & $5.3 b \pm 1.7$ & $5.6 \mathrm{ab} \pm 1.3$ \\
\hline Extruded Banana & $2.51 \mathrm{a} \pm 0.03$ & $3604 d \pm 558$ & $1.02 \mathrm{a} \pm 0.02$ & $0.34 a \pm 0.03$ & $0.16 b \pm 0.01$ & $45.87 \mathrm{a} \pm 0.52$ & $21.21 b \pm 0.76$ & $4.2 \mathrm{a} \pm 2.0$ & $6.2 b \pm 1.4$ & $5.5 b \pm 1.6$ & $4.7 \mathrm{a} \pm 1.8$ & $5.4 a \pm 1.5$ \\
\hline
\end{tabular}


Table 2. Degree of starch gelatinization and amylopectin retrogradation of bread crumb and crust.

\begin{tabular}{cccccc}
\hline & \multicolumn{2}{c}{ Crumb } & & \multicolumn{2}{c}{ Crust } \\
\cline { 2 - 3 } & Degree of starch & & Degree of starch & \\
& gelatinization during baking, & $\Delta \mathrm{H}_{\mathrm{r}}(\mathrm{J} / \mathrm{g})$ & & gelatinization during & $\Delta \mathrm{H}_{\mathrm{r}}(\mathrm{J} / \mathrm{g})$ \\
& $\mathrm{DG}_{\mathrm{B}}(\%)$ & & baking, $\mathrm{DG}_{\mathrm{B}}(\%)$ & \\
\hline Control & $100 \mathrm{a} \pm 0.00$ & $2.79 \mathrm{a} \pm 0.13$ & & $7.16 \mathrm{~b} \pm 0.56$ & n.d. \\
Native Banana & $100 \mathrm{a} \pm 0.00$ & $3.01 \mathrm{a} \pm 0.08$ & & $5.56 \mathrm{a} \pm 0.74$ & n.d. \\
Mixture Banana & $100 \mathrm{a} \pm 0.00$ & $3.38 \mathrm{~b} \pm 0.07$ & & $7.47 \mathrm{bc} \pm 0.55$ & n.d. \\
Extruded Banana & $100 \mathrm{a} \pm 0.00$ & $3.47 \mathrm{~b} \pm 0.16$ & & $8.48 \mathrm{c} \pm 0.99$ & n.d.
\end{tabular}

Values followed by the same letters within each parameter indicate no significant differences ( $\mathrm{p} \leq 0.05)$. n.d. non detectable.

$\Delta \mathrm{H}_{\mathrm{r}}$, enthalpy for retrograded amylopectin. 
Figure 1. Particle size distribution (A) of starchy ingredients and SEM pictures of native and extruded banana samples (B). Black circles highlight aggregates of banana starch granules.

A)

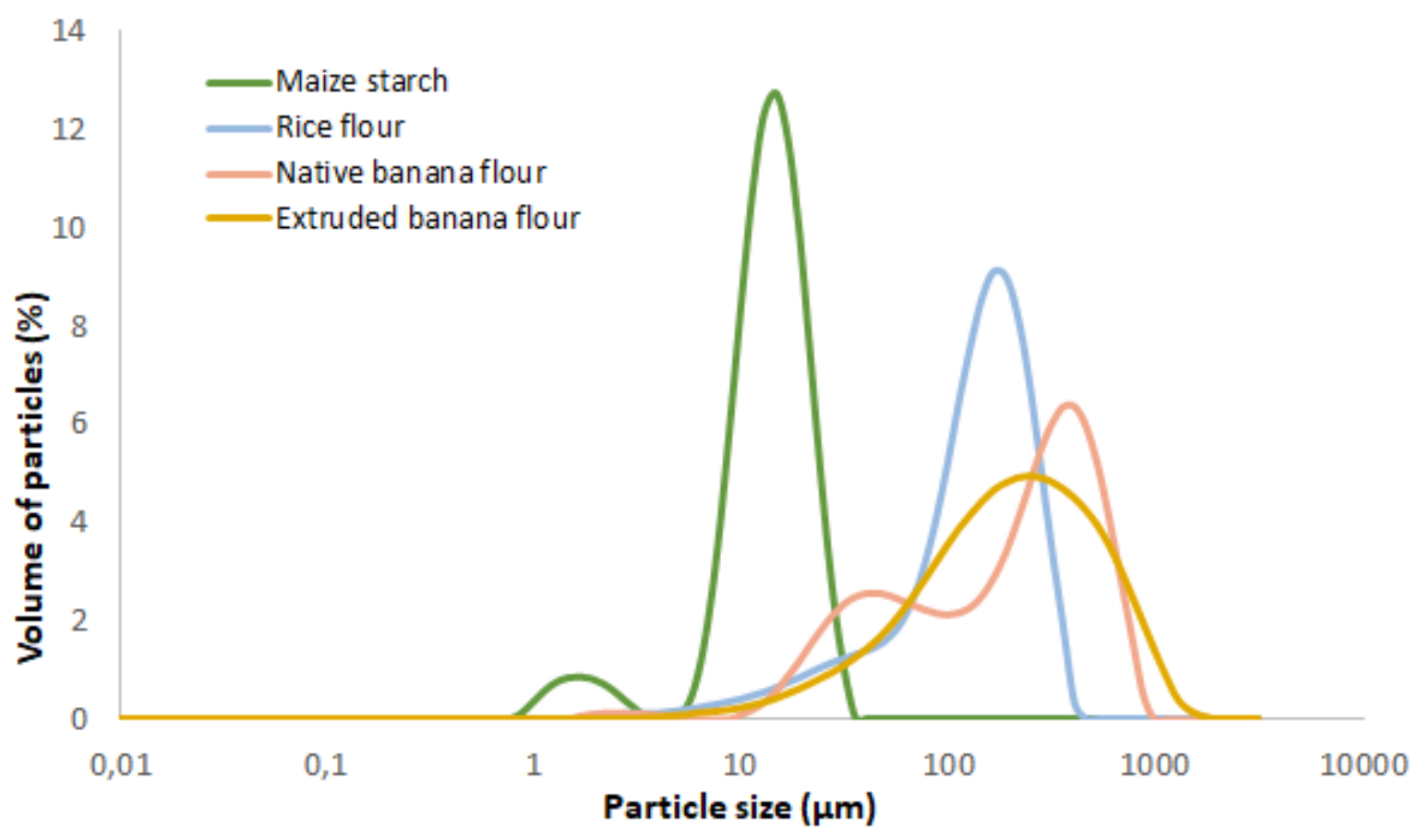


B)

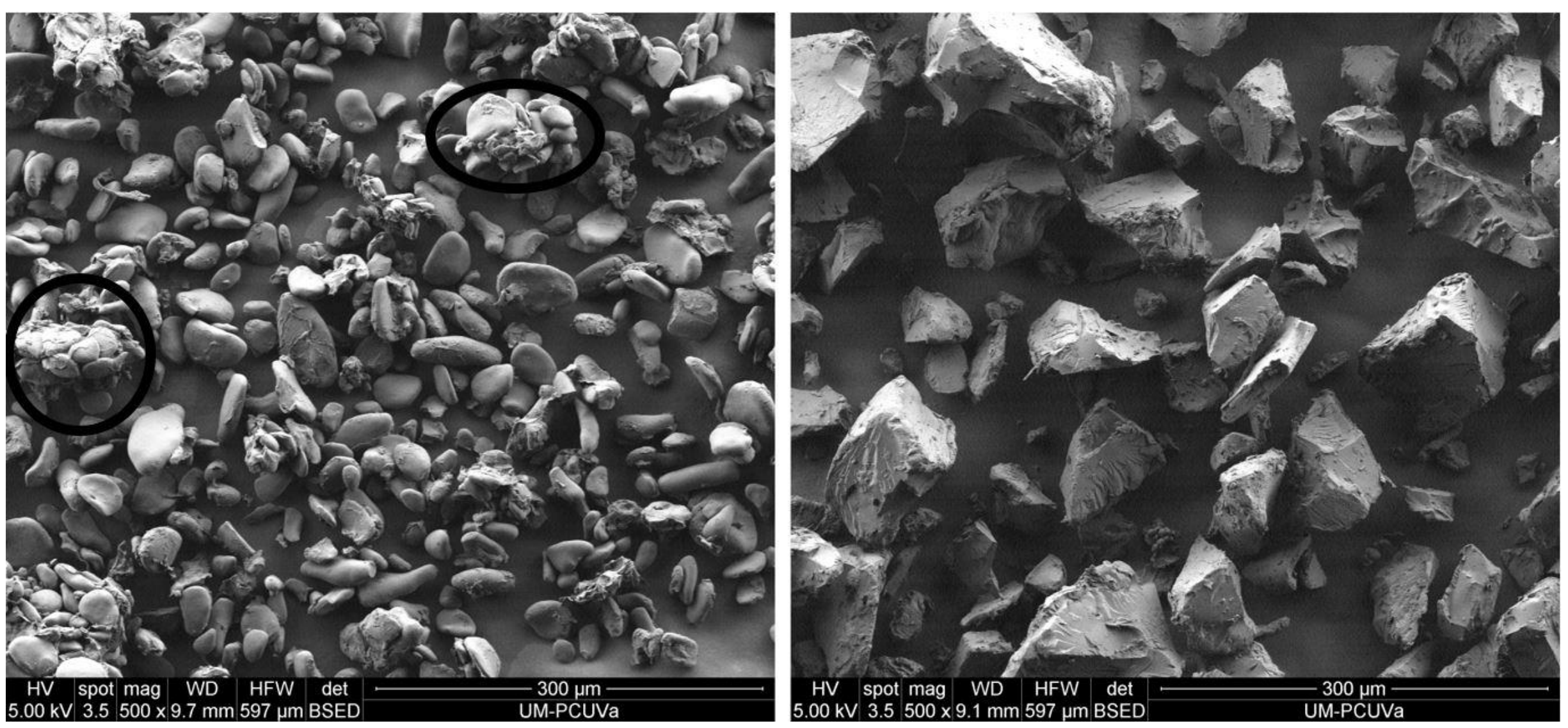


Figure 2. HPSEC-MALS chromatogram of native and extruded banana starch.

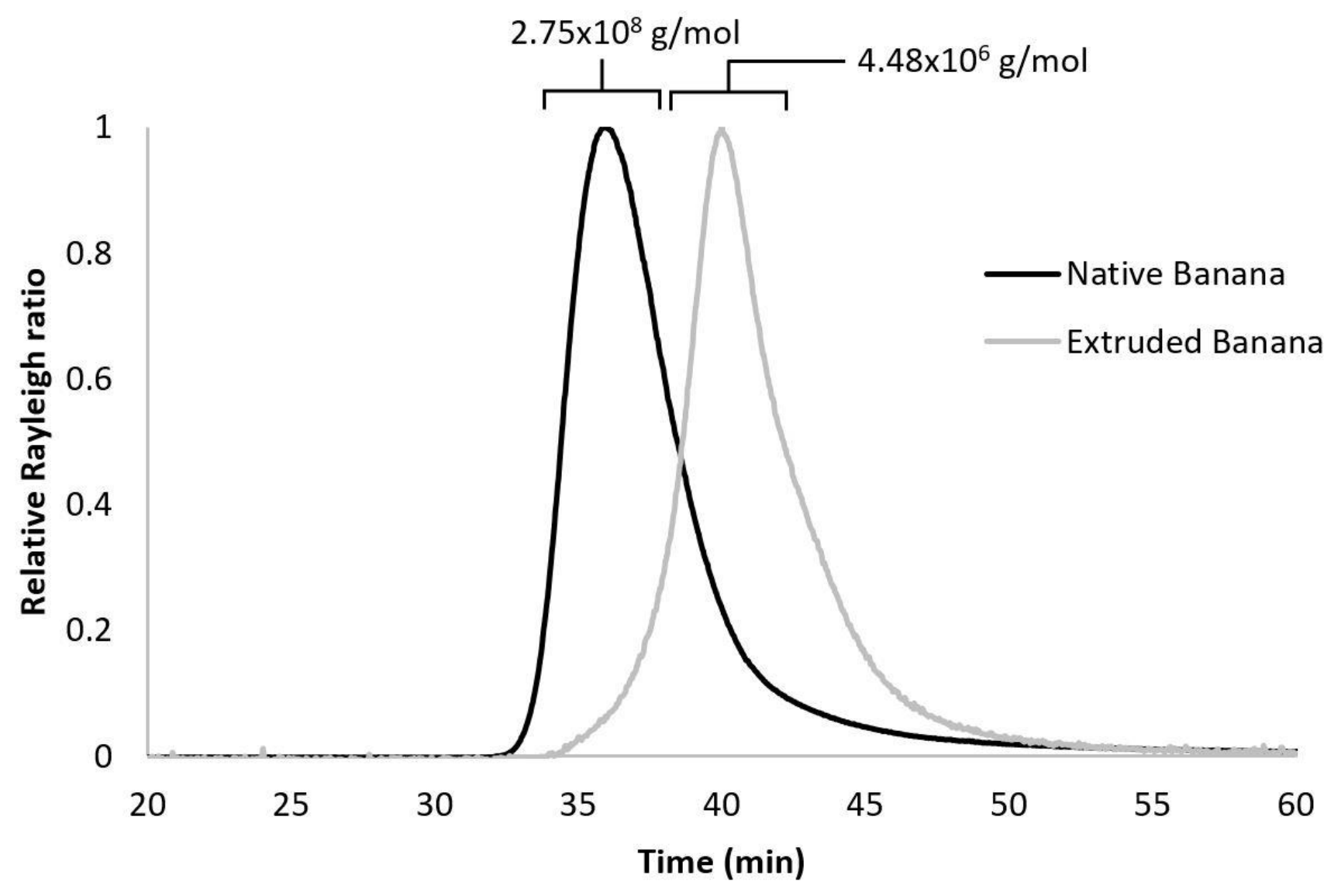


Figure 3. Pictures of central slices of bread. a) Control bread; b), bread made with $20 \%$ native banana starch (NB); c) bread made with 20\% of a 1:1 mixture of native and extruded banana starch (MB) and d) bread made with $20 \%$ extruded banana starch (EB).

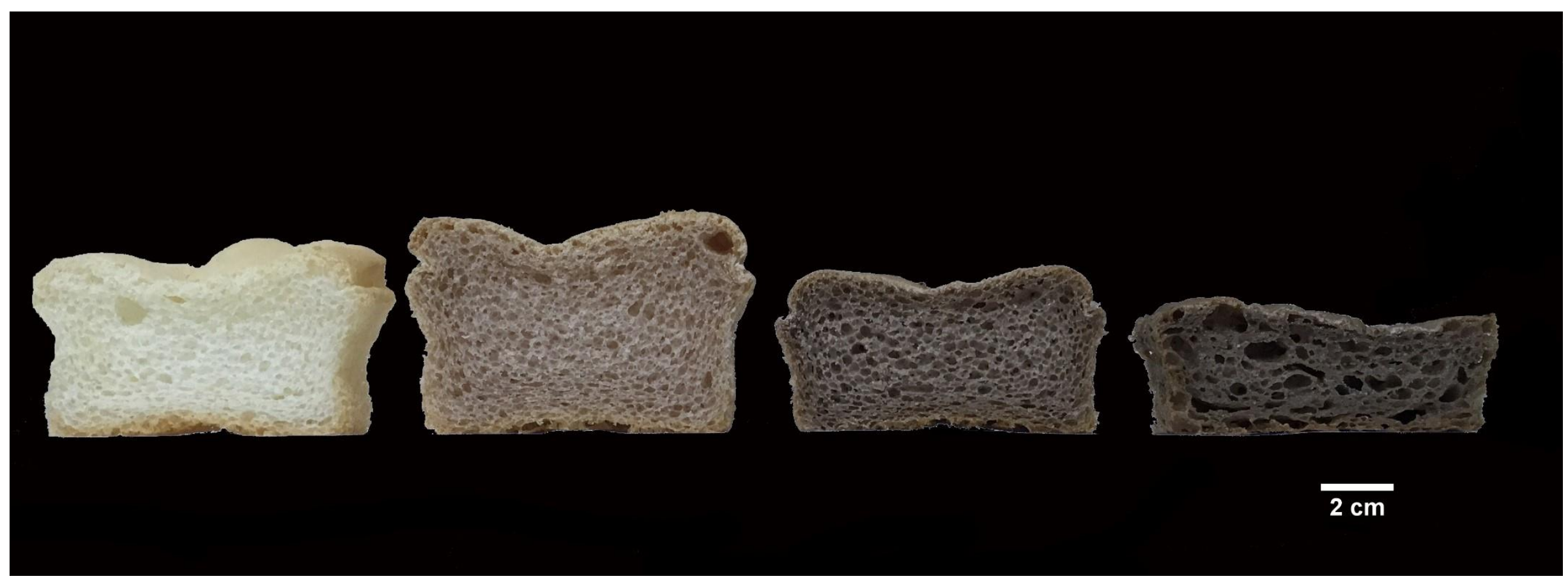


Figure 4. Slowly digestible starch (SDS) and resistant starch (RS) of bread crumb (left) and crust (right). a) Control bread; b), bread made with $20 \%$ native banana starch $(\mathrm{NB})$; c) bread made with $20 \%$ of a 1:1 mixture of native and extruded banana starch (MB) and d) bread made with $20 \%$ extruded banana starch (EB).
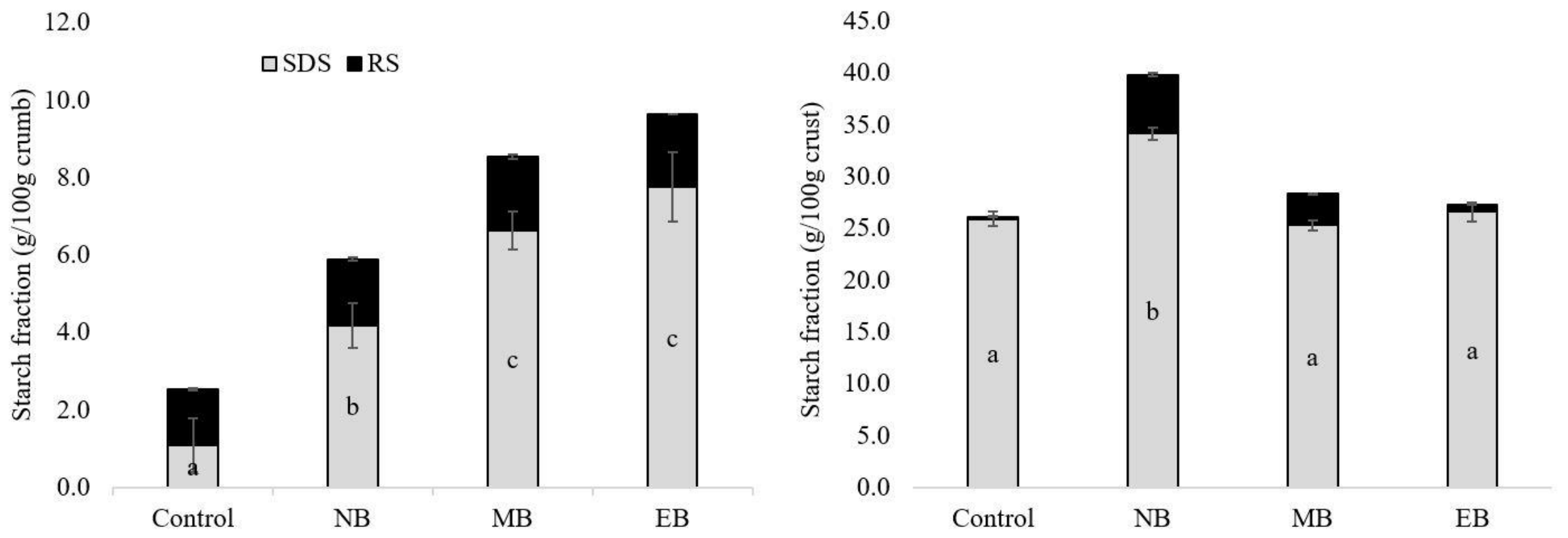


\section{Supplementary material}

Supplementary material 1. Gelatinization properties of starchy ingredients.

\begin{tabular}{lcccc}
\hline Sample & $\mathrm{T}_{\mathrm{o}}\left({ }^{\circ} \mathrm{C}\right)$ & $\mathrm{T}_{\mathrm{p}}\left({ }^{\circ} \mathrm{C}\right)$ & $\mathrm{T}_{\mathrm{c}}\left({ }^{\circ} \mathrm{C}\right)$ & $\Delta \mathrm{H}_{\mathrm{g}}(\mathrm{J} / \mathrm{g}, \mathrm{db})$ \\
\hline Rice flour & $67.57 \pm 0.02$ & $73.72 \pm 0.05$ & $81.64 \pm 0.20$ & $11.91 \pm 0.18$ \\
Maize starch & $62.12 \pm 0.14$ & $69.87 \pm 0.01$ & $85.36 \pm 0.60$ & $7.39 \pm 0.17$ \\
Native banana starch & $71.74 \pm 0.25$ & $78.62 \pm 0.03$ & $87.69 \pm 0.30$ & $10.81 \pm 0.19$ \\
Extruded banana starch & n.d. & n.d. & n.d. & n.d. \\
\hline
\end{tabular}

$T_{0}, T_{p}$ and $T_{c}$ indicates onset, peak and conclusion temperature of gelatinization; $\Delta H_{g}$, enthalpy of gelatinization expressed in $\mathrm{J} / \mathrm{g}$ dry solids, n.d. non detectable. 
Supplementary material 2. Pasting profile of starches and flours used for bread making.

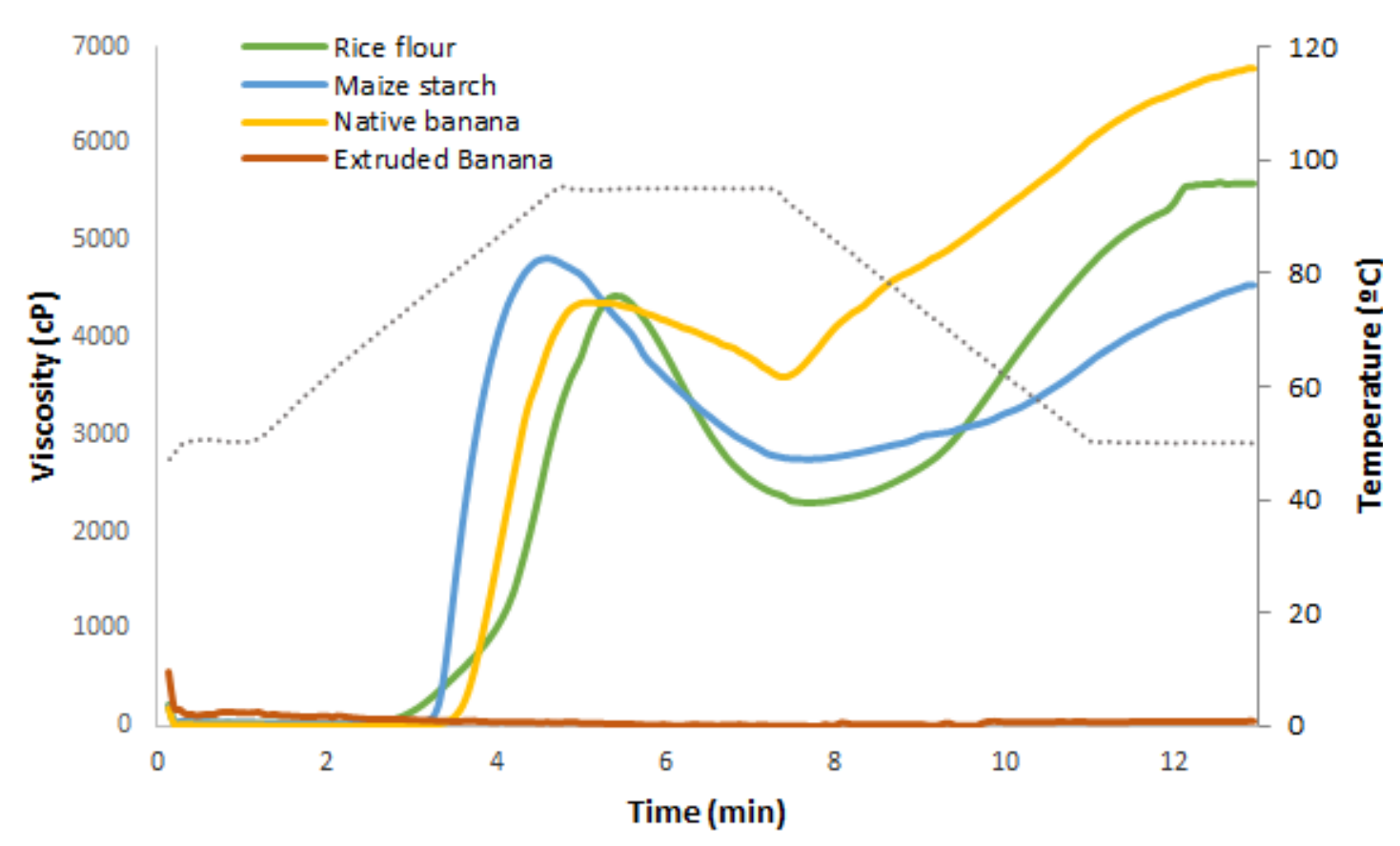


